\title{
Factores de riesgo prenatales en la muerte fetal tardía, Hospital Civil de Guadalajara, México
}

\author{
J. Guadalupe Panduro B. 1,3, J. Jesús Pérez M. 2,3, Elizabeth Guadalupe Panduro \\ M. ${ }^{1}$, Juan Francisco Castro H. 2,3, María Dolores Vázquez G. 1
}

1 Unidad de Medicina Materno Fetal, División de Ginecología y Obstetricia, Hospital Civil de Guadalajara "Dr. Juan I. Menchaca", 2 División de Pediatría, Hospital Civil de Guadalajara; ${ }^{3}$ Departamento de Reproducción Humana, Crecimiento y Desarrollo Infantil, Centro Universitario de Ciencias de la Salud, Universidad de Guadalajara. Guadalajara, Jalisco, México.

\section{RESUMEN}

Objetivo: Identificar los factores de riesgo sociodemográficos, obstétricos y perinatales que con más frecuencia se asocian a muerte fetal en embarazos mayores de 27 semanas. Método: De enero de 2004 a junio de 2009 en el Hospital Civil de Guadalajara, se realizó un estudio de casos y controles con 528 casos de muerte fetal de más de 27 semanas de gestación y 528 neonatos vivos cuyo nacimiento ocurrió inmediatamente después. Se comparó la frecuencia de diferentes variables maternas y fetales que en forma previa se han reportado asociadas a muerte fetal, por medio de Chi2 y prueba exacta de Fisher; se estimó la fuerza de asociación entre estas variables y muerte fetal con la razón de momios, con un intervalo de confianza del 95\%. Resultados: De los factores de riesgo estudiados se asociaron con muerte fetal: edad materna mayor de 35 años, escolaridad baja, multiparidad, antecedente de aborto y de muerte fetal, atención prenatal deficiente, complicaciones en el embarazo, líquido amniótico anormal, doble circular de cordón umbilical al cuello del producto y malformaciones congénitas mayores del recién nacido. No se asoció con muerte fetal, el estado civil soltero, ser primigesta, tabaquismo, sexo masculino del feto, circular simple al cuello y macrosomía fetal. Conclusiones: De los factores de riesgo asociados con muerte fetal, resalta la atención prenatal deficiente que de ser mejorada, podría disminuir la fuerza de asociación de algunas de las otras variables que se asociaron a muerte fetal.

\section{PALABRAS CLAVE: Muerte fetal, óbito fetal, atención prenatal, factores de riesgo}

\section{SUMMARY}

Objective: To identify sociodemographic, obstetric and perinatal factors most frequently associated with fetal death in pregnancies over 27 weeks. Methods: From January 2004 to June 2009 at the Civil Hospital of Guadalajara, we performed a case-control study of 528 stillbirths over 27 weeks gestation and 528 living infants whose birth occurred immediately afterwards. We compared the frequency of maternal and fetal variables that previously have been reported associated with fetal death by means of Chi2 and Fisher exact test, we estimated the strength of association between these variables and fetal death with odds ratios with a confidence level of $95 \%$. Results: The studied risk factors associated with fetal death was: maternal age older than 35 years, low schooling, multiparity, history of abortion and stillbirth, poor prenatal care, pregnancy complications, abnormal amniotic fluid, circular double umbilical cord around the neck of product and major 
congenital malformations of the newborn. Single marital status, primiparity, smoking, male fetus, simple circular neck and fetal macrosomia, was not associated with fetal death. Conclusions: Risk factors associated with fetal death, like poor prenatal care emphasizes that, if improved, could decrease the strength of association of some of the other variables associated with fetal death.

\section{KEY WORDS: Fetal death, stillbirth, prenatal care, risk factors}

\section{INTRODUCCIÓN}

En las últimas décadas, la obstetricia ha tenido avances importantes en el desarrollo de técnicas para evaluar el bienestar fetal, lo que nos hizo pensar que disminuiría en forma importante las tasas de mortalidad perinatal, sin embargo, siguen presentándose estos desafortunados desenlaces en el embarazo (1).

En la mayoría de casos de muerte fetal la causa es desconocida, siendo los problemas más frecuentemente asociados: la anoxia intrauterina, las malformaciones congénitas y la prematurez (2). La muerte fetal es más frecuente en las madres que no reciben atención prenatal adecuada, por lo que debe haber un mayor énfasis en la atención prenatal (3). Los factores que se han considerado más importantes desde el punto de vista fetal son anomalías cromosómicas, fetos pretérminos, bajo peso, sexo masculino y embarazo gemelar $(4,5)$. La causa de la muerte fetal permanece desconocida en la mitad de los casos, encontrándose como factor común en estos casos la restricción del crecimiento fetal (6).

Debido a que la muerte fetal constituye un problema serio en materia de salud y origina un impacto muy importante en las personas involucradas, repercutiendo importantemente en el ambiente familiar (7) se planeó realizar este estudio para evaluar los factores de riesgo sociodemográficos, obstétricos y perinatales que incrementan la mortalidad fetal tardía en la población que acude al Hospital Civil de Guadalajara, para que una vez detectados puedan ser de utilidad para disminuir la mortalidad fetal en futuros embarazos.

\section{MATERIAL Y MÉTODO}

Se utilizó un diseño de casos y controles. La población de estudio fueron los nacimientos ocurridos en el Hospital Civil de Guadalajara, "Dr. Juan I. Menchaca", de enero de 2004 a junio de 2009. Este Hospital brinda atención médica a población abierta, sin seguridad social, siendo en su mayoría de estrato socioeconómico bajo. En este periodo hubo 65.233 nacimientos, de los cuales $913(1,4 \%)$ fueron muertes fetales tanto tempranas como tardías. De este subgrupo, 547 correspondían a muertes fetales de embarazos mayores de 27 semanas $(0,8 \%)$.

La muestra de estudio se constituyó por 528 casos de muerte fetal tardía que correspondían a embarazos mayores de 27 semanas o peso $\geq 750$ gramos y un grupo control de 528 madres que tuvieron un recién nacido vivo mayor de 27 semanas o peso $\geq 750$ gramos y que su nacimiento haya ocurrido inmediatamente después del óbito. La muestra inicial había sido de 547 muertes fetales tardías, pero se eliminaron 19 casos, por no tener los datos completos del expediente, siendo excluidos también sus respectivos controles.

En la evaluación de los factores de riesgo la variable dependiente fue la muerte fetal y las variables independientes fueron la edad de las madres, estado civil, ocupación, escolaridad, tabaquismo, número de gestaciones, antecedente de abortos y de muerte fetal, control prenatal, edad gestacional, días sin movimientos fetales, complicaciones del embarazo, tipo de presentación y forma de nacimiento, características del líquido amniótico, presencia de circular de cordón o de nudo de cordón umbilical, sexo, peso y malformaciones del recién nacido.

Para estudiar las muertes fetales ocurridas durante el periodo de estudio se revisaron los libros de registro de los nacimientos del servicio de tococirugía; una vez localizados los registros de los óbitos, se tomó el registro correspondiente al siguiente nacimiento del caso, procediendo a revisar los expedientes clínicos y obtener las variables de estudio.

Las variables fueron capturadas en una hoja de cálculo en el programa Excel de Microsoft 2007 y analizadas con el programa SPSS versión 10.

Las variables cualitativas fueron contrastadas con la prueba Chi2 y exacta de Fisher. La asociación entre factores de riesgo y muerte fetal se midió por la razón de momios (RM) u Odds Ratio (OR), 
con intervalo de confianza del 95\% (IC95\%). Se consideró que había asociación cuando la RM fue mayor a uno y el intervalo de confianza no incluyó la unidad pero fue mayor que ésta.

La investigación fue aprobada por los comités de investigación y ética del Hospital Civil de Guadalajara "Dr. Juan I. Menchaca" con registro 679/026.

\section{RESULTADOS}

Se reportan los resultados de 1056 binomios materno fetales, los cuales se integraron en dos grupos: el grupo estudio con 528 casos de muerte fetal y el grupo control con 528 recién nacidos vivos que fueron seleccionados para participar en este estudio.

En relación con las variables sociodemográficas, la edad promedio materna fue 25,6 años para el grupo estudio, contra 23,9 años del grupo control. La frecuencia de madres mayores de 35 años fue mayor en el grupo de casos (61/528 vs $21 / 528$; $p<0,001)$; también, esta variable se asoció con mayor mortalidad fetal tardía (RM: 3,15; IC95\%: 1,84$5,44)$. El estado civil de las madres fue similar en ambos grupos (Tabla I).

En relación a la ocupación, se observó que las madres del grupo de casos se dedicaban con mayor frecuencia al hogar (509/528 vs 485/528; $p=0,001)$, (RM: 2,38; IC95\%: 1,32-4,29); se registró una menor escolaridad en el grupo de casos, evidenciado en el grado de primaria o menos (343/528 vs 259/528; $p<0,001$ ), (RM: 1,93; IC95\%: 1,49-2,48). El tabaquismo durante el embarazo fue similar en ambos grupos.

En las variables obstétricas, el promedio de gestaciones fue 2,9 en el grupo estudio y 2,7 en el grupo control, observando que el número de multigestas (4 ó más embarazos) fue mayor en el grupo de casos $(p=0,004)$, (RM: 1,48; IC95\%: 1,11-1,95), no siendo diferentes el número de primigestas. El antecedente de aborto fue mas frecuente en el grupo estudio, siendo también evidente el antecedente de dos o más abortos (13/528 vs $3 / 528 ; p=0,01)$, (RM: 4,42; IC95\%: 1,17-19,61) (Tabla II).

El antecedente de muerte fetal previa fue más frecuente en el grupo estudio (25/528 vs $3 / 528$; $\mathrm{p}<0,001)$, (RM: 8,70; IC95\%: 2,49-36,36). El antecedente de atención prenatal deficiente (ninguna a 2 consultas) también fue más frecuente en el grupo estudio (188/528 vs 65/528; $p<0,001)$, (RM: 3,94; IC 95\%: 2,84-5,47).

La edad gestacional del grupo estudio varió de 27 a 42 semanas, con promedio de 34,3 semanas, contra 38,7 semanas del grupo control. En relación a los días sin percibir los movimientos fetales, 198 madres $(37,5 \%)$ refirieron 24 horas o más sin percibir movimientos fetales, incluso 59 madres $(11,1 \%)$ tenían tres o más días sin movimientos fetales.

En relación a las complicaciones en el embarazo, en el grupo estudio 256 pacientes presentaron alguna complicación, como: amenaza de aborto, hipertensión arterial, infección urinaria, diabetes, patología placentaria y problemas tiroideos, mientras en el grupo control 135 pacientes presentaron alguna complicación, siendo las principales: amenaza de aborto, amenaza de parto pretérmino, infección de vías urinarias, rotura prematura de membranas, hipertensión arterial y diabetes (RM: 2,74; IC95\%: $2,10-3,58 ; p<0,001)$, encontrando diferencias significativas al comparar hipertensión arterial, diabetes y desprendimiento de placenta que fueron más frecuentes en el grupo estudio.

La presentación cefálica al nacimiento fue menor en el grupo de casos (401/528 vs 499/528), ya que los fetos muertos al ser pretérminos, es más frecuente la presentación pélvica. El nacimiento por cesárea fue $23,8 \%$ en el grupo estudio, contra $31,8 \%$ del grupo control $(p=0,003)$.

Tabla I

VARIABLES SOCIODEMOGRÁFICAS Y FUERZA DE ASOCIACIÓN CON MUERTE FETAL TARDÍA

\begin{tabular}{lccccc}
\hline Factores de riesgo & $\begin{array}{c}\text { Casos } \\
\mathrm{n}=528\end{array}$ & $\begin{array}{c}\text { Controles } \\
\mathrm{n}=528\end{array}$ & Valor $\mathrm{p}$ & $\begin{array}{c}\text { Razón de } \\
\text { Momios }\end{array}$ & IC 95\% \\
\hline Edad mayor de 35 años & 61 & 21 & $<0,001$ & 3,15 & $1,84-5,44$ \\
Casadas o con pareja & 436 & 429 & 0,575 & 1,09 & $0,79-1,51$ \\
Ocupación hogar & 509 & 485 & 0,001 & 2,38 & $1,32-4,29$ \\
Escolaridad s primaria & 343 & 259 & $<0,001$ & 1,93 & $1,49-2,48$ \\
Tabaquismo & 33 & 45 & 0,157 & 0,72 & $0,44-1,17$ \\
\hline
\end{tabular}


Tabla II

FACTORES OBSTÉTRICOS Y FUERZA DE ASOCIACIÓN CON MUERTE FETAL TARDÍA

\begin{tabular}{lccccc}
\hline Factores de riesgo & $\begin{array}{c}\text { Casos } \\
\mathrm{n}=528\end{array}$ & $\begin{array}{c}\text { Controles } \\
\mathrm{n}=528\end{array}$ & Valor $\mathrm{p}$ & $\begin{array}{c}\text { Razón de } \\
\text { Momios }\end{array}$ & IC 95\% \\
\hline Primigestas & 169 & 152 & 0,255 & 1,16 & $0,89-1,53$ \\
Multigestas $(\geq 4)$ & 167 & 126 & 0,004 & 1,48 & $1,11-1,95$ \\
Antecedente de aborto & 115 & 82 & 0,009 & 1,51 & $1,09-2,10$ \\
Dos o más abortos & 13 & 3 & 0,011 & 4,42 & $1,17-19,61$ \\
Muerte fetal previa & 25 & 3 & $<0,001$ & 8,70 & $2,49-36,36$ \\
Consultas $\leq 2$ & 188 & 65 & $<0,001$ & 3,94 & $2,84-5,47$ \\
Complicaciones en el embarazo & 256 & 135 & $<0,001$ & 2,74 & $2,10-3,58$ \\
Hipertensión & 77 & 38 & $<0,001$ & 2,20 & $1,44-3,38$ \\
Diabetes & 34 & 7 & $<0,001$ & 5,12 & $2,15-12,78$ \\
Desprendimiento de placenta & 19 & 3 & $<0,001$ & 6,53 & $1,82-27,89$ \\
\hline
\end{tabular}

En relación a factores de riesgo perinatales, se obtuvo en el grupo estudio una mayor frecuencia de líquido meconial $(275 / 528$ vs $66 / 528 ; p<0,001)$, (RM: 7,61; IC95\%: 5,52-10,50), así como mayor frecuencia de líquido rojizo (75/528 vs 3/528; p<0,001), (RM: 28,97; IC95\%: 8,76-115,73). Hubo mayor frecuencia de oligohidramnios en el grupo estudio (137/528 vs $26 / 528$; $p<0,001$ ), (RM: 6,77 ; IC95\%: 4,28-10,77), como también el polihidramnios (31/528 vs 4/528; $p<0,001)$, (RM: 8,17 ; IC95\%: 2,73-27,46) (Tabla III).

La circular de cordón al cuello del feto o recién nacido fue similar en ambos grupos $(p=0,123)$, pero la presencia de doble o triple circular de cordón fue más frecuente en el grupo estudio (32/528 vs 7/528; $p<0,001$ ), (RM: 4,80; IC95\%: 2,01-12,04). El hallazgo de un nudo verdadero de cordón, aunque se encontró en cinco fetos del grupo estudio contra uno del grupo control, no tuvo diferencia estadística $(p=0,101)$.

El sexo masculino fue más frecuente en el grupo estudio (291/528 vs $267 / 528)$ diferencia no significativa $(p=0,139)$. El peso de los recién nacidos fue menor en el grupo de casos, con promedio de $2.162 \mathrm{~g}$, contra $3.048 \mathrm{~g}$ del grupo control. Al comparar la macrosomía fetal asociada a diabetes materna se encontró diferencia estadística significativa $(p=0,025)$, al encontrarse 31 fetos macrosómicos del grupo estudio contra 16 del grupo control (Tabla III). Las malformaciones congénitas mayores del recién nacido, se presentaron en 35 de los fetos muertos, contra cuatro del grupo control (RM: 9,30; IC95\%: 3,13-31,03; $p<0,001)$. Las principales malformaciones fueron: hidrocefalia, gastrosquisis, labio paladar hendido, anencefalia, hidrops fetalis y malformaciones múltiples.

Tabla III

FACTORES PERINATALES Y FUERZA DE ASOCIACIÓN CON MORTALIDAD FETAL TARDÍA

\begin{tabular}{lccccc}
\hline Factores de riesgo & $\begin{array}{c}\text { Casos } \\
\mathrm{n}=528\end{array}$ & $\begin{array}{c}\text { Controles } \\
\mathrm{n}=528\end{array}$ & Valor $\mathrm{p}$ & $\begin{array}{c}\text { Razón de } \\
\text { Momios }\end{array}$ & IC 95\% \\
\hline Líquido amniótico meconial & 275 & 66 & $<0,001$ & 7,61 & $5,52-10,50$ \\
Líquido rojizo & 75 & 3 & $<0,001$ & 28,97 & $8,76-115,73$ \\
Oligohidramnios & 137 & 26 & $<0,001$ & 6,77 & $4,28-10,77$ \\
Polihidramnios & 31 & 4 & $<0,001$ & 8,17 & $2,73-27,46$ \\
Prolapso de cordón & 7 & 1 & 0,03 & 7,08 & $0,88-153,67$ \\
Circular de cordón & 95 & 115 & 0,123 & 0,79 & $0,58-1,08$ \\
Doble o triple circular & 32 & 7 & $<0,001$ & 4,80 & $2,01-12,04$ \\
Nudo del cordón & 5 & 1 & 0,101 & 5,04 & $0,57-114,35$ \\
Sexo masculino & 291 & 267 & 0,139 & 1,20 & $0,94-1,54$ \\
Peso del recién nacido $>$ 4000 g & 31 & 16 & 0,025 & 2,00 & $1,04-3,87$ \\
Malformaciones clínicas & 35 & 4 & $<0,001$ & 9,30 & $3,13-31,03$ \\
\hline
\end{tabular}




\section{DISCUSIÓN}

La muerte fetal, a pesar de que es un problema grave de salud pública con serias repercusiones en la vida familiar, no se le da la importancia debida, como lo deja de manifiesto este estudio en el que se evalúan 528 pacientes con muerte fetal comparándolos con 528 casos de madres con recién nacidos vivos, en el que a través de dos parámetros observamos la poca importancia que se tiene en la vigilancia prenatal, como es el que 188 pacientes del grupo de estudio (35,6\%) asistieron en 0,1 ó máximo 2 ocasiones a control prenatal, lo que deja de manifiesto la escasa vigilancia en estas pacientes, así mismo 198 madres $(37,5 \%)$ refirieron tener 24 horas o más sin percibir movimientos fetales, incluso 59 madres $(11,1 \%)$ tenían 3 o más días sin movimientos fetales, al momento de presentarse al hospital en donde se les hizo el diagnóstico de muerte fetal.

El antecedente de muerte fetal es muy importante, ya que en este estudio se encontró una diferencia significativa, al observar $25(4,7 \%)$ casos contra $3(0,6 \%)$ del grupo control, habiéndose reportado previamente por Frias y cols (4), quienes señalan que existe un pobre pronóstico obstétrico en embarazos subsecuentes en mujeres que han tenido una muerte fetal. Esto es más importante si consideramos que en este estudio el $32,0 \%$ de las pacientes del grupo de casos eran primigestas y quienes seguramente tendrán después nuevos embarazos, por lo que estas mujeres deben tener una vigilancia más estrecha, con un control prenatal adecuado para evitar en lo posible un desenlace fatal del siguiente embarazo.

La edad gestacional promedio en el grupo de casos fue significativamente menor (34,3 vs 38,7 semanas), al igual que lo reportado por Romero y cols (8), quienes en su serie de 250 muertes fetales, reportan que un $70,4 \%$ correspondía a embarazos menores de 37 semanas, lo que justifica el mayor número de presentaciones no cefálicas $(24,2 \%$ vs $5,5 \%$ ) que se observan con más frecuencia en embarazos pretérminos.

La vía de terminación del embarazo fue más frecuente vaginal en el grupo estudio $(76,1 \%)$ que el grupo control $(68,1 \%)$, con diferencia significativa, $(p=0,003)$. Aunque los embarazos pretérmino eran más frecuentes en el grupo estudio en quienes el cérvix no está favorable para un parto, la mayoría de las pacientes acuden al hospital hasta que ya tienen contracciones uterinas, por eso se hacía un diagnóstico tardío, siendo esto favorable para que se pudieran dejar a nacimiento por vía vaginal siendo menor el índice de cesáreas en este grupo. En ocasiones se utiliza misoprostol vía vaginal ( 25 a 50 microgramos cada 4 a 6 horas) como lo ha reportado Gómez y cols (9) con lo que se reduce el número de cesáreas.

Los factores de riesgo que se han asociado a muerte fetal en otros estudios, que no tuvieron diferencia estadística significativa fueron: el estado civil, en donde por las características de la población estudiada, de bajos recursos que acuden al Hospital Civil de Guadalajara, solo un porcentaje menor $253(47,9 \%)$ del grupo estudio y $183(34,6 \%)$ del grupo control son casadas y que al sumarles el estado civil de unión libre, no tuvieron diferencia al compararlas con el estado civil soltero. Solo un mínimo porcentaje admitió fumar durante el embarazo $6,2 \%$ y $8,5 \%$ de cada grupo, no habiendo diferencias, contrario a lo reportado por Romero y cols (8) que aunque reportaron una frecuencia baja de 3,2 contra $0,4 \%$ si encuentra una diferencia estadística significativa. El grupo de primigestas, que fue ligeramente mayor en el grupo de estudio (32\% vs $28,8 \%)$, no tuvo diferencia significativa $(p=0,255)$. El circular de cordón, que popularmente se asocia a muerte fetal, en este estudio presentaron circular de cordón a cuello fetal, 95 recién nacidos muertos $(17,9 \%)$ vs 115 recién nacidos del grupo control $(21,7 \%)$, lo que no tuvo diferencia estadística significativa $(p=0,123)$, que coincide con lo reportado por Wang y cols (10). El sexo masculino fetal, aunque fue más frecuente en el grupo estudio (291 vs 267), no presentó diferencia significativa $(p=0,139)$. Se encontró asociación significativa entre muerte fetal y macrosomía como ha sido reportado por Mondestin y cols (11), independiente de diabetes materna.

Los factores de riesgo que se asociaron a muerte fetal tardía fueron: edad mayor de 35 años, ocupación hogar, escolaridad baja, multiparidad, antecedente de aborto, antecedente de muerte fetal, atención prenatal deficiente, complicaciones en el embarazo, alteraciones en líquido amniótico, doble o triple circular de cordón umbilical a cuello, macrosomía fetal y malformaciones clínicas mayores en el neonato, coincidentes con múltiples publicaciones.

La principal limitación de nuestro estudio es que no incluye resultados de autopsia, lo cual es debido a la cultura de la población mexicana que solo permite estudios de autopsia en casos de medicina legal, dificultando así el conocer las causas verdaderas de muerte fetal, lo que aunque no era objetivo de este trabajo hubiera sido importante por el número de muertes fetales estudiadas.

En relación a los factores de riesgo asociados a muerte fetal tardía, es importante hacer mención, que la vigilancia prenatal tiene un gran impacto en la disminución de la muerte fetal, ya que si acudiera la paciente a consulta prenatal se podrían determi- 
nar factores de riesgo para desarrollar preeclampsia, diabetes y amenaza de parto pretérmino, así como con el ultrasonido se podrían diagnosticar alteraciones del líquido amniótico, presencia de doble circular de cordón, malformaciones fetales y alteraciones del crecimiento fetal. Además al identificar otros factores de riesgo como la edad mayor de 35 años, baja escolaridad, ocupación hogar, antecedente de aborto y antecedente de muerte fetal, se tendría que hacer una vigilancia prenatal más estrecha, así como se prestaría una especial atención en la educación prenatal para la prevención y detección precoz de posibles complicaciones que pudieran culminar con la muerte del feto.

\section{CONCLUSIÓN}

Se concluye que la muerte fetal tardía, se asocia a diversos factores de riesgo, siendo el principal, sobre el que giran los demás, la atención prenatal inadecuada, por lo que se sugiere mejorar la atención prenatal, con un mayor número de consultas distribuidas a lo largo del embarazo, otorgadas con calidad y calidez, prestando especial atención en los factores de riesgo, que de ser identificados y abordados de forma adecuada, quizá se pueda evitar una muerte fetal con toda la tragedia personal y familiar que esto representa.

\section{BIBLIOGRAFÍA}

1. American College of Obstetricians and Gynecologist. Management of stillbirth. ACOG Practice Bulletin No. 102. Obstet Gynecol 2009;113:748-61.
2. Sheiner E, Hallak M, Shoha-Vardi I, Goldstein D, Mazor M, Katz M. Determining risk factors for intrapartum fetal death. J Reprod Med 2000;45:419-24.

3. De Aquino MM, Cecatti JG, Mariani Neto C. Risk factors associated to fetal death. Sao Paulo Med J 1998;116:1852-7.

4. Frias AE, Luikenaar RA, Sullivan AE, Lee RM, Porter TF, Branch DW, Silver RM. Poor obstetric outcome in subsequent pregnancies in women with prior fetal death. Obstet Gynecol 2004;104:521-6.

5. Kahn B, Lumey LH, Zybert PA, Lorenz JM, ClearyGoldman J, Dalton ME, Robinson JN. Prospective risk of fetal death in singleton, twin, and triplet gestations: implications for practice. Obstet Gynecol 2003;102:685-92.

6. Gardosi J, Badger S, Tonks A, Francis A. Unexplained stillbirths: An investigation of the clinically relevant conditions at the time of fetal death. Am J Obstet Gynecol 2003;189:158s.

7. Gold KJ, Kuznia AL, Hayward RA. How physicians cope with stillbirth or neonatal death: a national survey of obstetricians. Obstet Gyneol 2008;112: 29-34.

8. Romero GG, Martínez CCA, Abrego OE, Ponce PAL. Multivariate analysis of risk factors for stillbirth in Leon, Mexico. Acta Obstet Gynecol Scand 2005;84:2-6.

9. Gómez Ponce de León R, Wing D, Fiala C. Misoprostol for intrauterine fetal death. Int J Gynecol Obstet 2007;99:s190-3.

10. Wang Y, Le Ray C, Audibert F, Wagner MS. Management of nuchal cord with multiple loops. Obstet Gynecol 2008;112(2 pt2):460-1.

11. Mondestin MAJ, Ananth CV, Smulian JC, Vintzileos AM. Birth weight and fetal death in the United States. The effect of maternal diabetes during pregnancy. Am J Obstet Gynecol 2002;187:922-6. 